\title{
Soxhtherm Extraction, Isolation and Identification of Fatty Acids Present in the Hexane Extract of Abutilon Pannosum and Grewia Tenax Using Gas Chromatography-Mass Spectrometry
}

\author{
Mital K. Aadesariya, Vijay R. Ram, Pragnesh N. Dave* \\ Department of Chemistry, KSKV Kachchh University, Bhuj, Kachchh- 370001
}

*Corresponding Author: Pragnesh N. Dave, Department of Chemistry, KSKV Kachchh University, Bhuj, Kachchh-370001

\begin{abstract}
Plants have been significant source of drugs with potential for thousands of years. Phytochemicals are the chemicals extracted from plants. These organic chemicals are classified as primary or secondary constituents, depending on their role in plant metabolism. ${ }^{[1]}$ GC-MS method used for the analysis of the obtained extract can be an interesting tool for testing the amount of some active principles in herbs used in various industries. ${ }^{[2]}$ The main objective of the study was to evaluate the fatty acids composition for identified bioactive compound in leaf hexane extract of the Abutilon pannosum and Grewia tenax was analysed by gas chromatography combined with Mass Spectrometry. While the mass spectra of the compounds found in the extract was matched with the National Institute of Standards and Technology (NIST) library. Fatty acids play a crucial role in growth and development of the body. It is known to have antibacterial and antifungal properties. ${ }^{[3]}$ The shade dried both plant leaf powder was extracted with hexane by using Gerhardt Soxhtherm extractor and crude hexane extract was obtained. Derivatization was performed and then gas chromatography-mass spectrometry (GC-MS) was done for detecting fatty acids. From that eleven phytochemical constituents have been identified. The GC-MS analysis revealed the presence of various compounds like 9, 12-Octadecadienoic, 9-Octadecenoic acid, Hexadecanoic acid, Octadecanoic acid, Octadecatrienoic acid, 9, 12, 15-Eicosanoic acid, 9-Hexadecenoic acid, 11-Eicosenoic acid, Methyl tetradecanoate, Octanoic acid and Tridecanoic acid. These findings support the traditional use of A. pannosum and $G$. tenax in various disorders.
\end{abstract}

Keywords: Fatty acid, GC-MS, Abutilon pannosum and Grewia tenax

\section{INTRODUCTION}

Abutilon pannosum and Grewia tenax is an important medicinal plant in the Indian system of Medicine. It is commonly called khapat or kanghi and gangeti or gudaim, which grows in warm and arid regions. A. pannosum is used in cleaning wound and ulcer, treating vaginal infection, diabetics, haemorrhoids and can also use as an anaemia. ${ }^{[4]} G$. tenax is used tonsillitis, bone fracture and swelling, lactation, anaemia, porridge. ${ }^{[5]}$ Gas Chromatography Mass Spectroscopy, a hyphenated system which is a very compatible technique and the most commonly used technique for the identification and quantification purpose. The unknown organic compounds in a complex mixture can be determined by interpretation and also by matching the spectra with reference spectra. ${ }^{[6]}$ Fatty acids are widely occurring in natural fats and dietary oils, and they are also important nutritious substances and metabolites in living organisms. ${ }^{[7]}$ The human body needs essential fatty acids to construct and repair cell membranes enabling the cells to obtain optimum nutrition and expel harmful waste products. ${ }^{[8]} \mathrm{A}$ primary function of essential fatty acids, which support the cardiovascular, reproductive, immune and nervous systems, is the production of prostaglandins. ${ }^{[9]}$ These regulate body functions such as heart rate, blood pressure, blood clotting, fertility and play a role in immune system by regulating inflammation. ${ }^{[10-12]}$ Yet there is no report about the fatty acid composition of hexane extract leaves of A. pannosum and G. tenax species, which is the subject of the present study.

\section{Materials AND Methods}

\subsection{Extraction Method}

About $20 \mathrm{~g}$ of powdered material of aerial parts of two selected plants (A. pannosum and G. tenax) were extracted with $750 \mathrm{~mL} \mathrm{n}$-hexane for six hours through Gerhardt soxtherm apparatus. The 
extracts were concentrated by recovering the solvent using rotary evaporator. The next step was derivatization of the fatty acids in order to make them volatile to be capable of being analysed with gas chromatography-mass spectrometry (GC-MS). Methylation is the most general method of converting non-volatile fatty acids into volatile fatty acids methyl esters.

\subsection{Derivatization Method}

First $100 \mathrm{mg}$ of extract was weighed in $250 \mathrm{ml}$ Round bottom flask and kept it on a heating mantle at $45^{\circ} \mathrm{C}$. After that $4 \mathrm{ml}$ methanolic $\mathrm{NaOH}$ was added and boiled for 5 minutes. Next $2 \mathrm{ml}$ Boron trifluoride was added and boiled it for 5 minutes. Then heating was stopped and $4 \mathrm{ml} \mathrm{Hexane} \mathrm{was} \mathrm{added}$ and round bottom flask (r.b.f.) was removed from the heating mantle and $15 \mathrm{ml}$ saturated Nacl was added allow r.b.f. to cool and mixture was transferred to a test tube for phase separation. After that 0.5-1 gm sodium sulphate was taken in the eppendrof and the upper phase was transferred into it and vortex for 2 minutes for moisture and water removal and last $1.5 \mathrm{ml}$ of oil is transferred into GC vial. [13]

\subsection{GC-MS Method}

The plant extract samples were analysed using Shimadzu GC-2010 system comprising an AOC-20i auto-sampler and interfaced to a Mass Spectrometer (QP Plus 2010) equipped with a DB-wax (100\% Poly-Ethylene Glycol, polar fused capillary column $(30 \times 0.25 \mu \mathrm{m}$ ID $\times 0.25 \mu \mathrm{m} \mathrm{df})$. For GC-MS detection, an electron ionization system was operated in Electron Impact (EI) mode with ionization energy of $70 \mathrm{eV}$. Helium gas (99.999\%) was used as a carrier gas at a constant flow rate of $1 \mathrm{ml} / \mathrm{min}$, and an injection volume of $1 \mu \mathrm{l}$ was employed (a split ratio of 50:1). The injector temperature was maintained at $250^{\circ} \mathrm{C}$, the ion-source temperature was $230^{\circ} \mathrm{C}$, the oven temperature was programmed from $60^{\circ} \mathrm{C}$ with an increase of $12^{\circ} \mathrm{C} / \mathrm{min}$ to $150^{\circ} \mathrm{C}$ (isothermal for $1 \mathrm{~min}$ ), then the oven temperature was increased at a rate of $5^{\circ} \mathrm{C} / \mathrm{min}$ to $240^{\circ} \mathrm{C}$ (isothermal for $5 \mathrm{~min}$ ), Mass spectra were taken at 70 $\mathrm{eV}$; a scan interval of $0.5 \mathrm{sec}$ and fragments from $50 \mathrm{~m} / \mathrm{z}$ to $1000 \mathrm{~m} / \mathrm{z}$. The solvent delay was 0 to 2.30 $\mathrm{min}$, and the total GC/MS running time was $31.50 \mathrm{~min}$.

\subsection{Identification Method}

Identification of the fatty acid methyl ester was conducted by comparing the mass spectrum with NIST library. The compounds showing more that 90\% Similarity Index (SI) was identified and recorded further characterization. The bioactivities of the identified compounds related to medicinal and chemical property were identified from online database of NIST, PubChem, PubMed and Chem Spider etc.

\section{RESULT AND DISCUSSION}

GC-MS chromatogram of the hexane extract of leaves of Abutilon pannosum (Fig. 1) clearly showed 11 peaks and G. tenax has showed 9 peaks that were indicating the presence of 11 and 9 phytochemical compounds respectively. The identification of the phytochemical compounds was founded on the peak area, retention time and molecular formula. The table 1 shows the compound name with its molecular formula, Retention time, Peak area and \% Peak area. The results reveal the presence of 9, 12-Octadecadienoic (45.05\%), 9-Octadecenoic acid (33.18\%), Hexadecanoic acid $(12.12 \%)$, Octadecanoic acid (5.88\%), Octadecatrienoic acid $(2.62 \%), 9,12,15-$ Eicosanoic acid $(0.60 \%)$, 9-Hexadecenoic acid (0.22\%), 11-Eicosenoic acid (0.16\%), Methyl tetradecanoate $(0.10 \%)$, Octanoic acid $(0.04 \%)$ and Tridecanoic acid $(0.03 \%)$ in hexane extract of A.pannosum and 9, 12Octadecadienoic $(48.50 \%)>$ 9-Octadecenoic acid $(32.02 \%)>$ Hexadecanoic acid $(11.48 \%)>$ Octadecanoic acid (6.20\%) > 9, 12, 15- Octadecatrienoic acid $(0.81 \%)>$ Eicosanoic acid $(0.56 \%)>9$ Hexadecenoic acid $(0.19 \%)>11$-Eicosenoic acid $(0.18 \%)$ and Methyl tetradecanoate $(0.06 \%)$ in hexane extract of G. tenax. The phytochemical compounds recognized through GC-MS analysis showed many biological activities are listed in Table 2. While the mass spectra of the compounds found in the extract was matched with the National Institute of Standards and Technology (NIST) library. The results obtained in the analyses of the hexane extract of Abutilon pannosum are listed in below Table 1, 
Soxhtherm Extraction, Isolation and Identification of Fatty Acids Present in the Hexane Extract of Abutilon Pannosum and Grewia Tenax Using Gas Chromatography-Mass Spectrometry

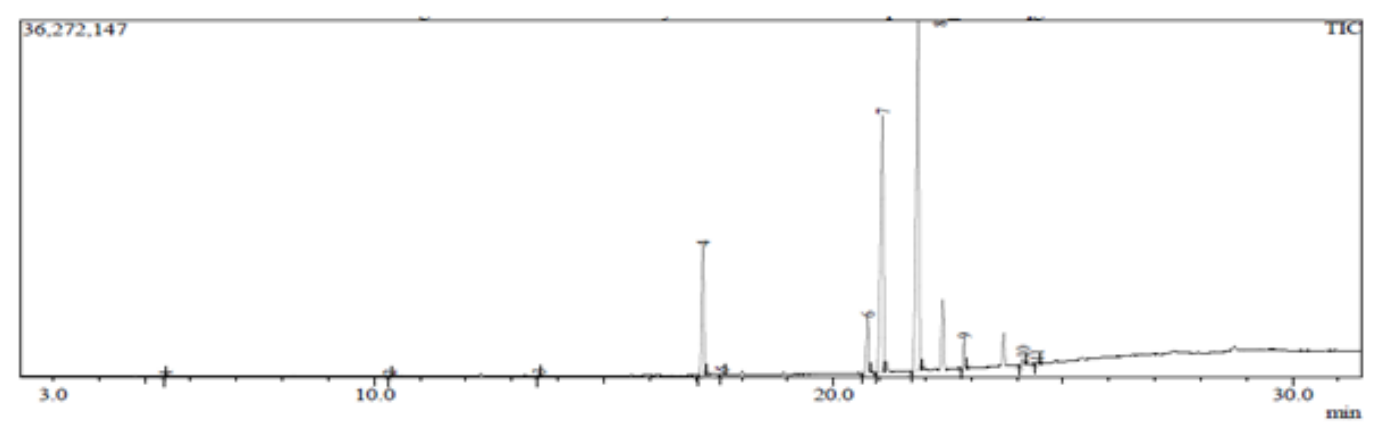

Figure1. The GC-MS Chromatogram of n-hexane extract of leaves of Abutilon pannosum

CompName:Octanoic acid, methyl ester

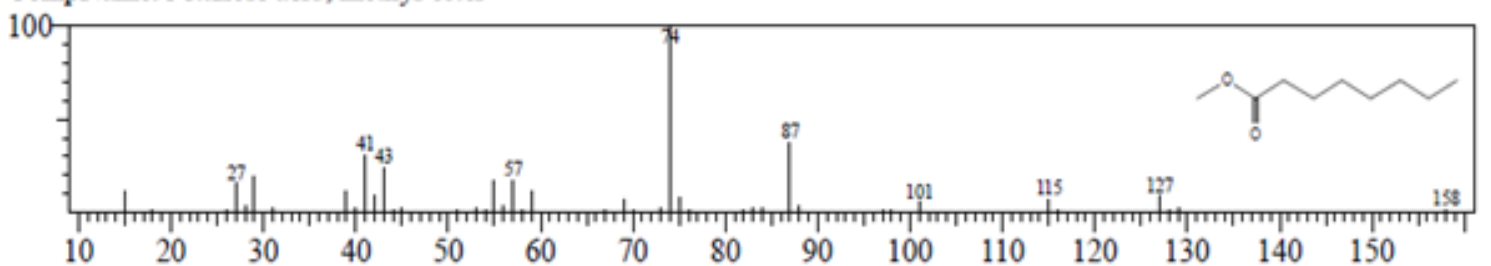

Figure2. Mass spectrum of Octanoic acid

CompName:Tridecanoic acid, methyl ester

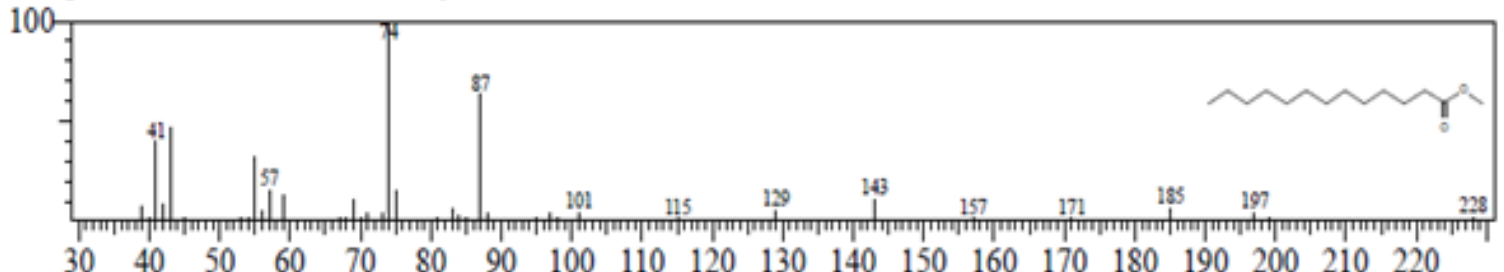

Figure3. Mass spectra of Tridecanoic acid

CompName:Methyl tetradecanoate

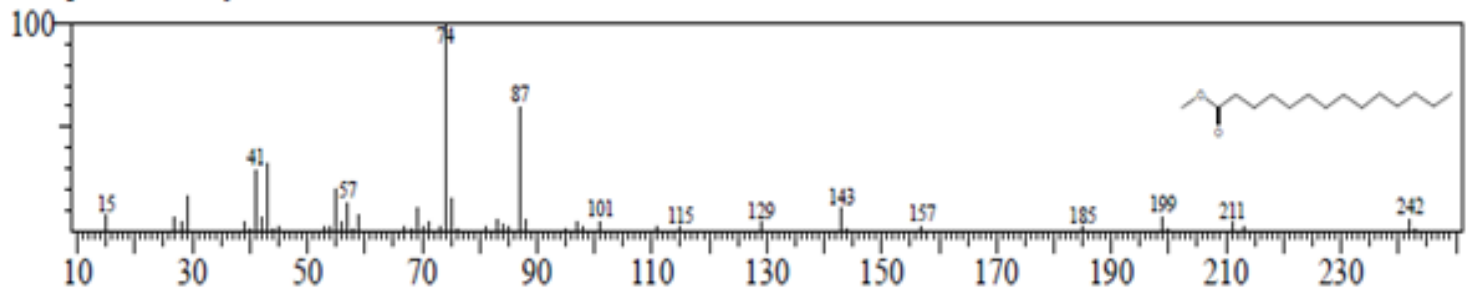

Figure4. Mass spectrum of Methyl tetradecanote

CompName:Hexadecanoic acid, methyl ester

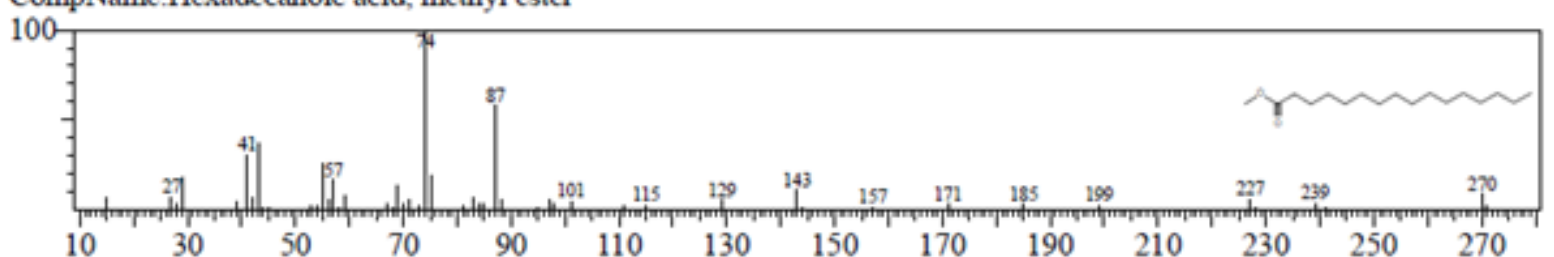

Figure5. Mass spectrum of Hexadecanoic acid

CompName:9-Hexadecenoic acid, methyl ester, (Z)- \$\$ Methyl palmitoleate \$\$ Methyl palmitoleinate \$ Palmitoleic acid,

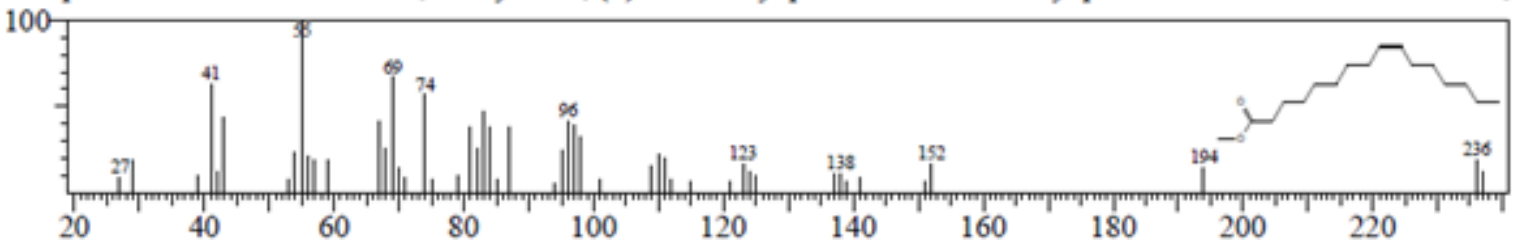

Fig6. Mass spectrum of 9-Hexadecanoic acid 
Soxhtherm Extraction, Isolation and Identification of Fatty Acids Present in the Hexane Extract of Abutilon Pannosum and Grewia Tenax Using Gas Chromatography-Mass Spectrometry

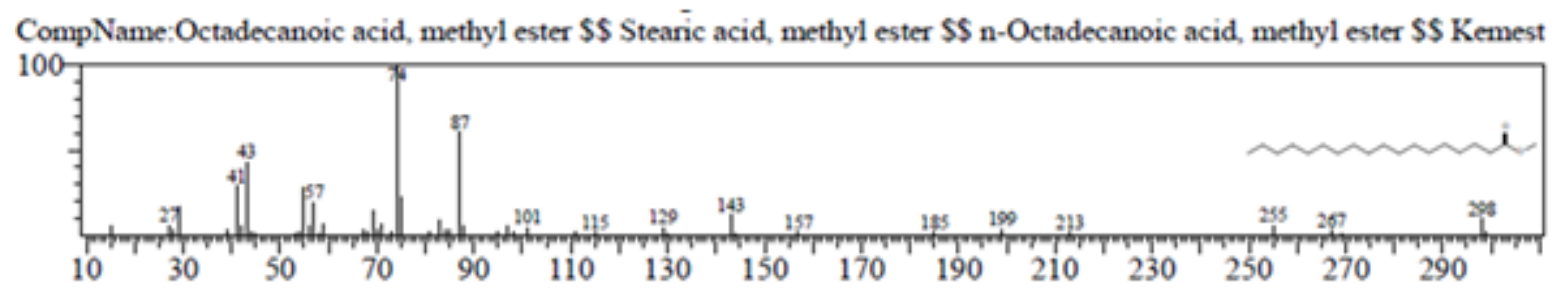

Fig7. Mass spectrum of Octadecanoic acid

CompName:9-Octadecenoic acid (Z)-, methyl ester

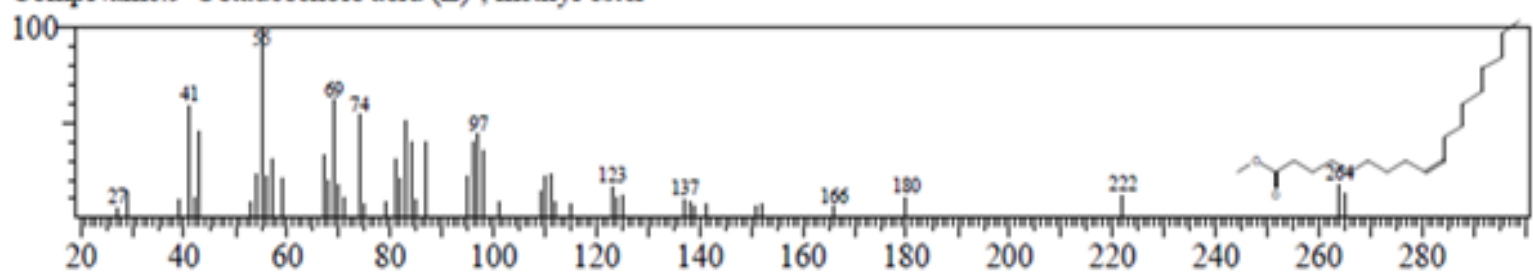

Fig8. Mass spectrum of 9-Octadecanoic acid (Z)

CompName:9,12-Octadecadienoic acid, methyl ester \$\$

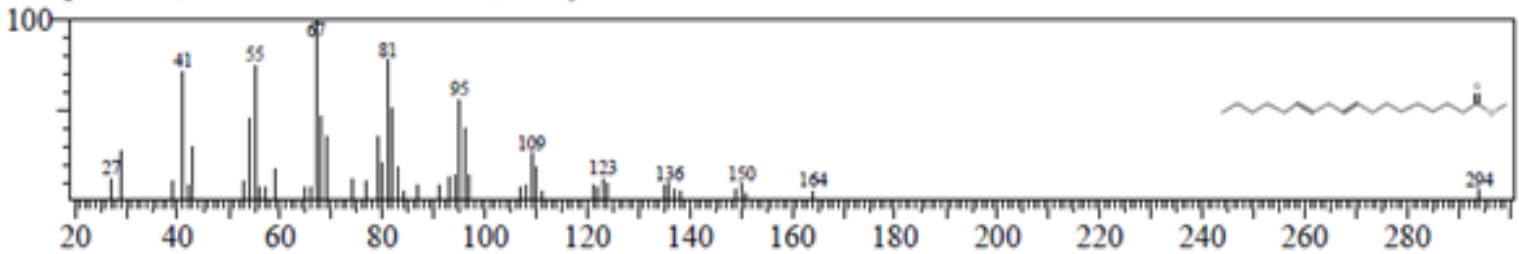

Fig9. Mass spectrum of 9, 12-Octadecanoic acid

CompName:9,12,15-Octadecatrienoic acid, methyl ester, (Z,Z,Z)- \$\$ Linolenic acid, methyl ester \$\$ Methyl all-cis-9,12,1

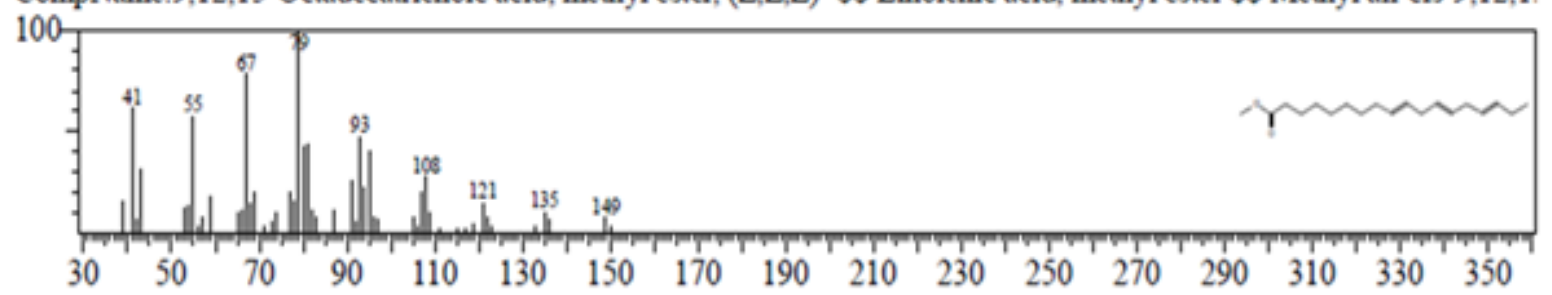

Fig10. Mass spectrum of 9, 12, 15-Octadecanoic acid

CompName:Eicosanoic acid, methyl ester \$\$ Methyl arachate \$\$ Methyl eicosanoate \$\$ Arachidic acid methyl ester \$\$

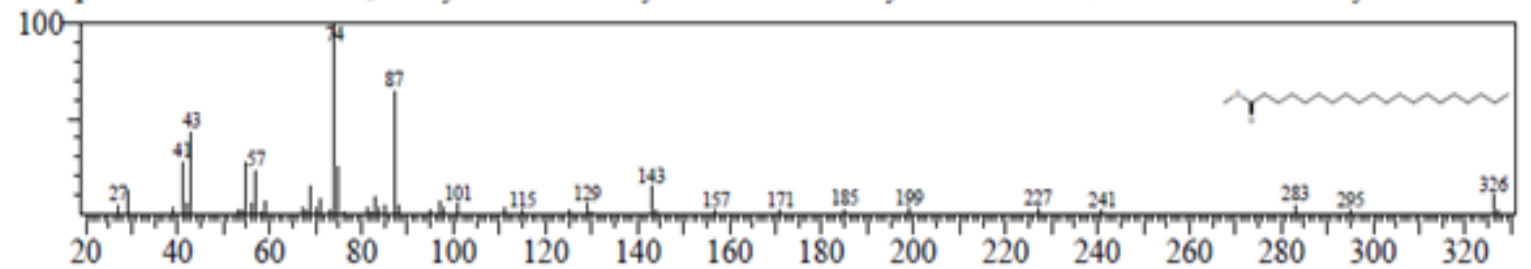

Fig11. Mass spectrum of Eicosanoic acid

CompName:11-Eicosenoic acid, methyl ester \$\$ Methyl (11E)-11-icosenoate \#\$\$

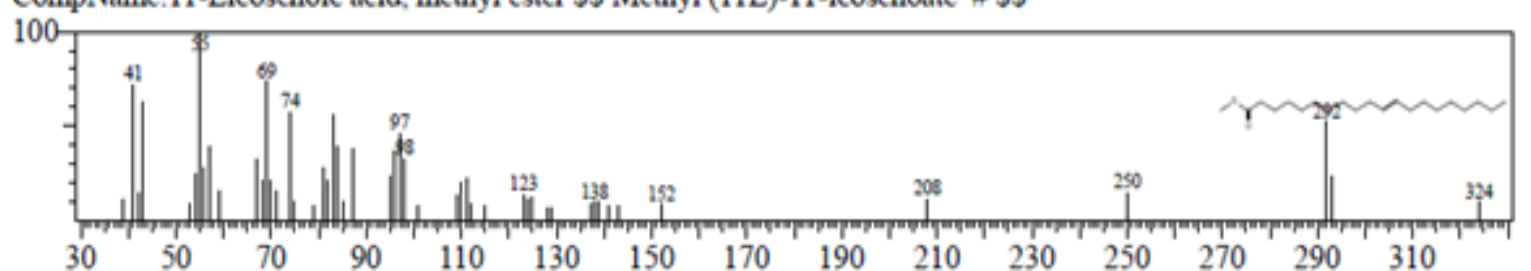

Fig12. Mass spectrum of 11-Eicosanoic acid 
Table1. List of Bioactive compound of hexane extract of leaf of Abutilon pannosum

\begin{tabular}{|c|c|c|c|c|c|c|c|c|c|c|}
\hline $\begin{array}{l}\text { Sr. } \\
\text { No }\end{array}$ & RT & $\begin{array}{c}\text { Area } \\
\%\end{array}$ & H \% & \begin{tabular}{c|}
$\mathrm{A} / \mathrm{H}$ \\
ratio
\end{tabular} & $\begin{array}{l}\text { Fatty Acid Methyl } \\
\text { Ester }\end{array}$ & M.F & CAD ID & $\begin{array}{l}\text { M. } \\
\text { W. }\end{array}$ & SI & $\begin{array}{l}\text { Libr } \\
\text { ary }\end{array}$ \\
\hline 1 & 5.443 & 0.04 & 0.11 & 1.53 & $\begin{array}{c}\text { Octanoic acid, methyl } \\
\text { ester }\end{array}$ & $\mathrm{C}_{9} \mathrm{H}_{18} \mathrm{O}_{2}$ & 111-11-5 & 158 & 94 & $\begin{array}{l}\text { Nist } \\
27\end{array}$ \\
\hline 2 & 10.331 & 0.03 & 0.06 & 1.90 & $\begin{array}{c}\text { Tridecanoic acid, } \\
\text { methyl ester }\end{array}$ & $\mathrm{C}_{14} \mathrm{H}_{28} \mathrm{O}_{2}$ & 1731-88-0 & 228 & 86 & $\begin{array}{c}\text { Nist2 } \\
7\end{array}$ \\
\hline 3 & 13.584 & 0.10 & 0.16 & 2.43 & Methyl tetradecanoate & $\mathrm{C}_{15} \mathrm{H}_{30} \mathrm{O}_{2}$ & 124-10-7 & 242 & 92 & $\begin{array}{l}\text { Nist } \\
27\end{array}$ \\
\hline 4 & 17.159 & 12.12 & 15.44 & 3.17 & $\begin{array}{l}\text { Hexadecanoic acid, } \\
\text { methyl ester }\end{array}$ & $\mathrm{C}_{17} \mathrm{H}_{34} \mathrm{O}_{2}$ & 112-39-0 & 270 & 97 & $\begin{array}{l}\text { Nist } \\
27\end{array}$ \\
\hline 5 & 17.569 & 0.22 & 0.28 & 3.10 & $\begin{array}{l}\text { 9-Hexadecenoic acid, } \\
\text { methyl ester, }(Z)\end{array}$ & $\mathrm{C}_{17} \mathrm{H}_{32} \mathrm{O}_{2}$ & $1120-25-8$ & 268 & 89 & $\begin{array}{l}\text { Nist } \\
147\end{array}$ \\
\hline 6 & 20.749 & 5.88 & 6.71 & 3.54 & $\begin{array}{c}\text { Octadecanoic acid, } \\
\text { methyl ester }\end{array}$ & $\mathrm{C}_{19} \mathrm{H}_{38} \mathrm{O}_{2}$ & 112-61-8 & 298 & 96 & $\begin{array}{l}\text { Nist } \\
147\end{array}$ \\
\hline 7 & 21.073 & 33.18 & 30.8 & 4.35 & $\begin{array}{l}\text { 9-Octadecenoic acid } \\
\text { (Z)-, methyl ester }\end{array}$ & $\mathrm{C}_{19} \mathrm{H}_{36} \mathrm{O}_{2}$ & 112-62-9 & 296 & 96 & $\begin{array}{l}\text { Nist } \\
27\end{array}$ \\
\hline 8 & 21.846 & 45.05 & 41.98 & 4.34 & $\begin{array}{l}\text { 9,12-Octadecadienoic } \\
\text { acid, methyl ester }\end{array}$ & $\mathrm{C}_{19} \mathrm{H}_{34} \mathrm{O}_{2}$ & $2462-85-3$ & 294 & 96 & $\begin{array}{l}\text { Nist } \\
107\end{array}$ \\
\hline 9 & 22.855 & 2.62 & 3.51 & 3.01 & $\begin{array}{c}9,12,15- \\
\text { Octadecatrienoic acid, } \\
\text { methyl ester, }(\mathrm{Z}, \mathrm{Z}, \mathrm{Z})-\end{array}$ & $\mathrm{C}_{19} \mathrm{H}_{32} \mathrm{O}_{2}$ & $301-00-8$ & 292 & 95 & $\begin{array}{l}\text { Nist } \\
107\end{array}$ \\
\hline 10 & 24.127 & 0.60 & 0.74 & 3.26 & $\begin{array}{l}\text { Eicosanoic acid, methyl } \\
\text { ester }\end{array}$ & $\mathrm{C}_{21} \mathrm{H}_{42} \mathrm{O}_{2}$ & 1120-28-1 & 326 & 96 & $\begin{array}{l}\text { Nist } \\
107\end{array}$ \\
\hline 11 & 24.431 & 0.16 & 0.21 & 3.01 & $\begin{array}{l}\text { 11-Eicosenoic acid, } \\
\text { methyl ester }\end{array}$ & $\mathrm{C}_{21} \mathrm{H}_{40} \mathrm{O}_{2}$ & $3946-08-5$ & 324 & 81 & $\begin{array}{l}\text { Nist } \\
147\end{array}$ \\
\hline
\end{tabular}

According to above results showed that total eleven type fatty acid present in n-hexane extract of $A$. pannosum. It was mainly found to be in order of 9, 12-Octadecadienoic (45.05\%) > 9-Octadecenoic acid $(33.18 \%)>$ Hexadecanoic acid $(12.12 \%)>$ Octadecanoic acid $(5.88 \%)>9,12,15$-Octadecatrienoic acid $(2.62 \%)>$ Eicosanoic acid $(0.60 \%)>9$-Hexadecenoic acid $(0.22 \%)>11$-Eicosenoic acid $(0.16 \%)>$ Methyl tetradecanoate $(0.10 \%)>$ Octanoic acid $(0.04 \%)$ and Tridecanoic acid $(0.03 \%)$.

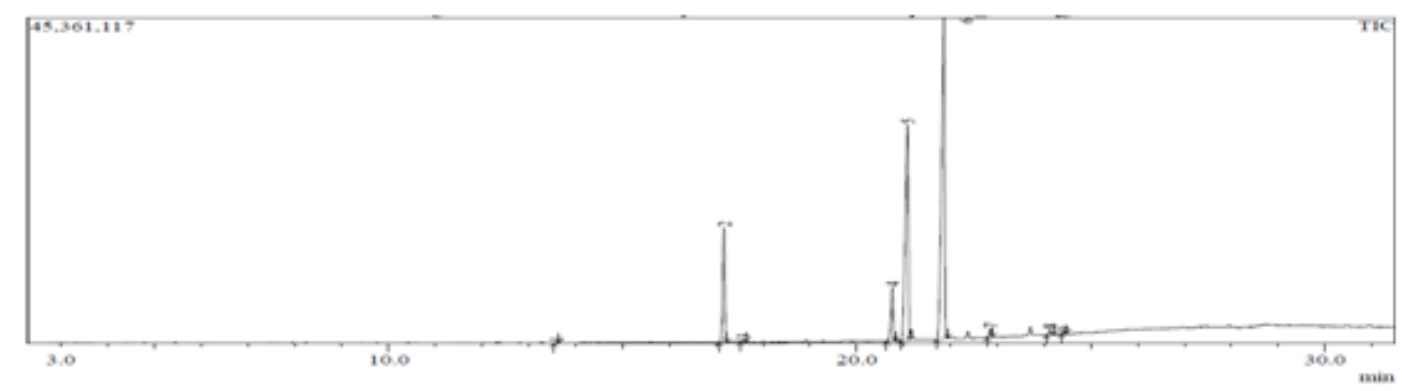

Figure13. GC - MS Chromatogram of n-hexane extract of leaves of Grewia tenax

CompName:Methyl tetradecanoate

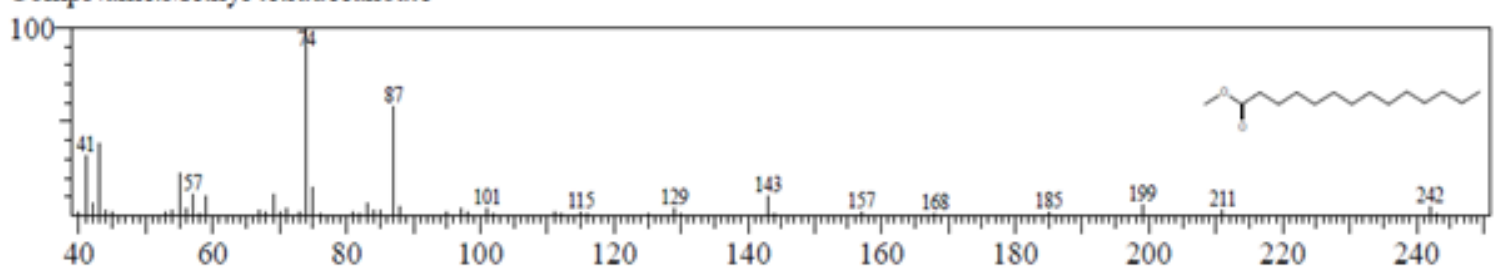

Figure14. Mass spectrum of Methyl tetradecanote

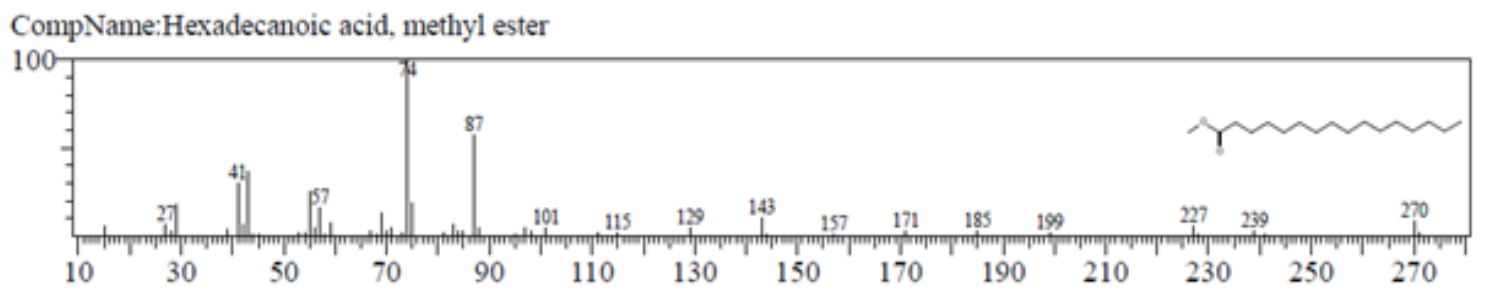

Figure15. Mass spectrum of Hexadecanoic acid 
Soxhtherm Extraction, Isolation and Identification of Fatty Acids Present in the Hexane Extract of Abutilon Pannosum and Grewia Tenax Using Gas Chromatography-Mass Spectrometry

CompName:9-Hexadecenoic acid, methyl ester, (Z)- \$\$ Methyl palmitoleate \$\$ Methyl palmitoleinate \$\$ Palmitoleic acid, 100

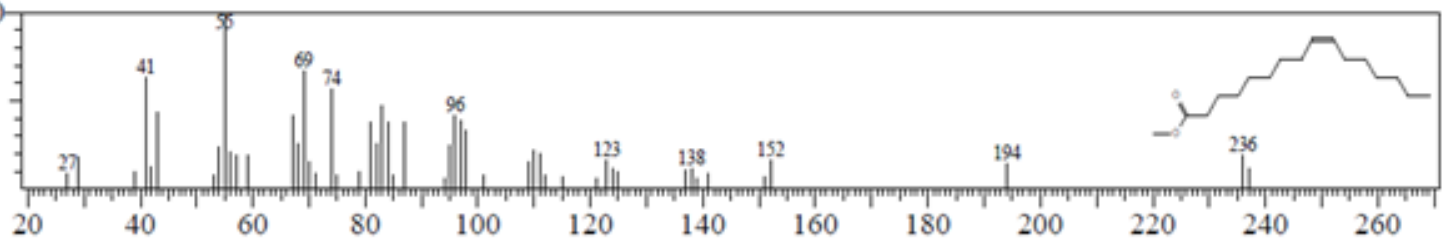

Figure16. Mass spectrum of 9-Hexadecanoic acid

CompName:Octadecanoic acid, methyl ester \$\$ \$tearic acid, methyl ester \$\$ n-Octadecanoic acid, methyl ester \$\$ Kemest 100

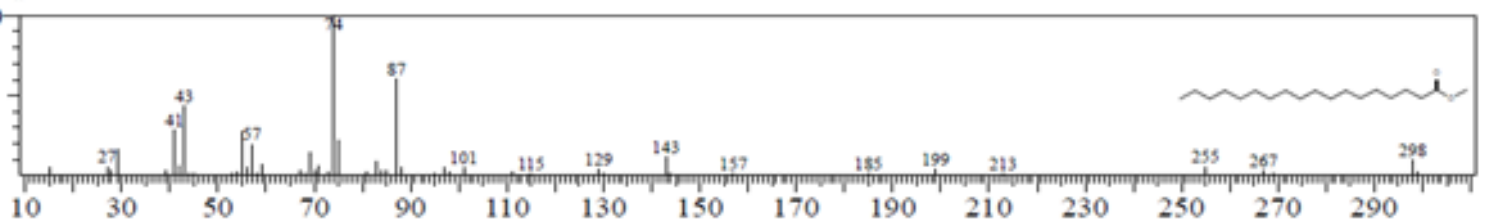

Figure17. Mass spectrum of Octadecanoic acid

CompName:9-Octadecenoic acid (Z)-, methyl ester $\$ \$$ Oleic acid, methyl ester $\$ \$$ Emery oleic acid ester $2301 \$ \$$ Methyl 100

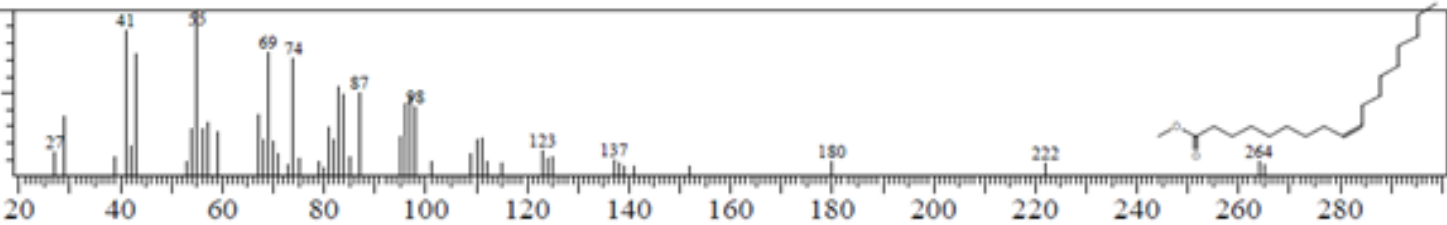

Figure18. Mass spectrum of 9-Octadecanoic acid

CompName:9,12-Octadecadienoic acid, methyl ester $\$ \$$

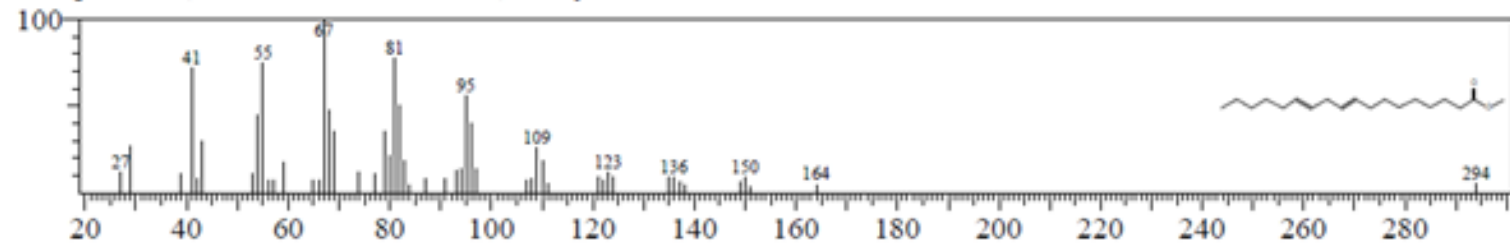

Figure19. Mass spectrum of 9, 12-Octadecanoic acid

CompName:9,12,15-Octadecatrienoic acid, methyl ester, (Z,Z,Z)- \$\$ Linolenic acid, methyl ester $\$ \$$ Methyl all-cis-9,12,1:

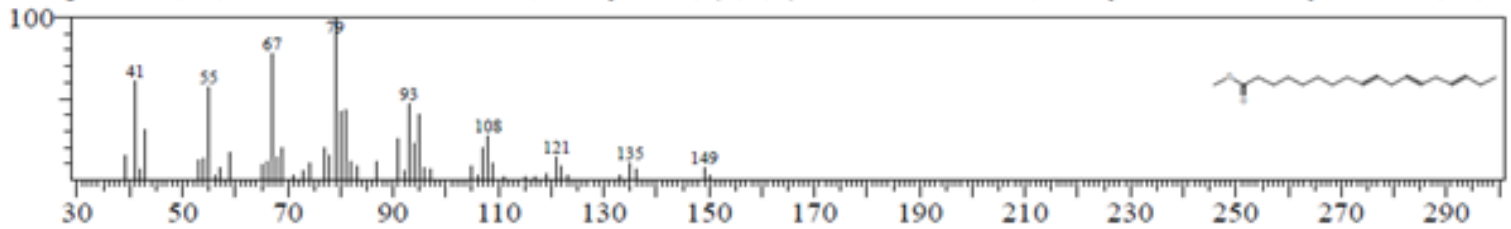

Figure20. Mass spectrum of 9, 12, 15-Octadecanoic acid

CompName:Eicosanoic acid, methyl ester \$\$ Methyl arachate \$\$ Methyl eicosanoate \$\$ Arachidic acid methyl ester \$\$ $100-$

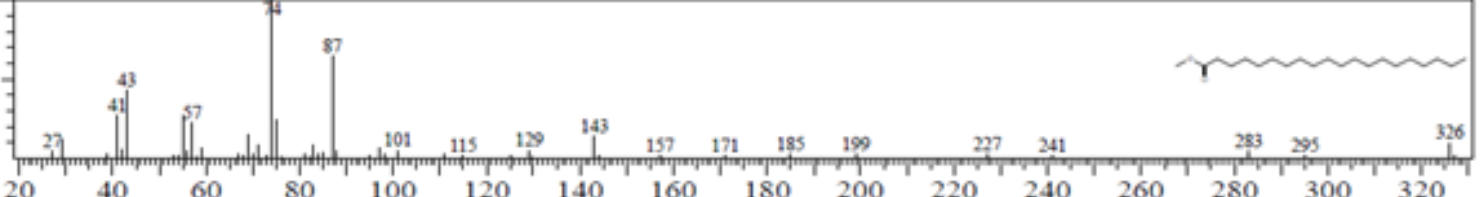

Figure21. Mass spectrum of Eicosanoic acid

CompName:11-Eicosenoic acid, methyl ester $\$ \$$

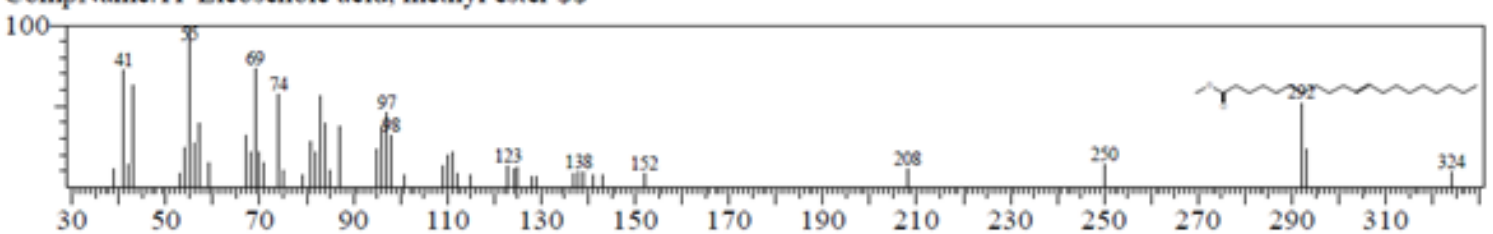

Figure22. Mass spectrum of 11- Eicosanoic acid 
Soxhtherm Extraction, Isolation and Identification of Fatty Acids Present in the Hexane Extract of Abutilon Pannosum and Grewia Tenax Using Gas Chromatography-Mass Spectrometry

Table2. List of Bioactive compound of hexane extract of leaf of Grewia tenax

\begin{tabular}{|c|c|c|c|c|c|c|c|c|c|c|}
\hline $\begin{array}{l}\text { Sr. } \\
\text { No }\end{array}$ & RT & $\begin{array}{c}\text { Area } \\
\%\end{array}$ & H \% & $\begin{array}{l}\mathrm{A} / \mathrm{H} \\
\text { ratio }\end{array}$ & $\begin{array}{c}\text { Fatty Acid } \\
\text { Methyl Ester }\end{array}$ & M.F & CAD ID & $\begin{array}{l}\text { M. } \\
\text { W }\end{array}$ & SI & Library \\
\hline 1 & 13.582 & 0.06 & 0.09 & 2.80 & $\begin{array}{l}\text { Methyl } \\
\text { tetradecanoate }\end{array}$ & $\mathrm{C}_{15} \mathrm{H}_{30} \mathrm{O}_{2}$ & $124-10-7$ & 242 & 92 & Nist27 \\
\hline 2 & 17.166 & 11.48 & 15.82 & 3.16 & $\begin{array}{l}\text { Hexadecanoic } \\
\text { acid, methyl } \\
\text { ester }\end{array}$ & $\mathrm{C}_{17} \mathrm{H}_{34} \mathrm{O}_{2}$ & $112-39-0$ & 270 & 97 & Nist27 \\
\hline 3 & 17.569 & 0.19 & 0.28 & 2.95 & $\begin{array}{l}\text { 9-Hexadecenoic } \\
\text { acid, methyl } \\
\text { ester, (Z)- }\end{array}$ & $\mathrm{C}_{17} \mathrm{H}_{32} \mathrm{O}_{2}$ & $1120-25-8$ & 268 & 89 & Nist147 \\
\hline 4 & 20.759 & 6.20 & 7.39 & 3.65 & $\begin{array}{l}\text { Octadecanoic } \\
\text { acid, methyl } \\
\text { ester }\end{array}$ & $\mathrm{C}_{19} \mathrm{H}_{38} \mathrm{O}_{2}$ & $112-61-8$ & 298 & 96 & Nist147 \\
\hline 5 & 21.086 & 32.02 & 29.8 & 4.68 & $\begin{array}{l}\text { 9-Octadecenoic } \\
\text { acid }(Z)-, \text { methyl } \\
\text { ester }\end{array}$ & $\mathrm{C}_{19} \mathrm{H}_{36} \mathrm{O}_{2}$ & $112-62-9$ & 296 & 96 & Nist27 \\
\hline 6 & 21.863 & 48.50 & 44.49 & 4.75 & $\begin{array}{l}\text { 9,12- } \\
\text { Octadecadienoic } \\
\text { acid, methyl } \\
\text { ester }\end{array}$ & $\mathrm{C}_{19} \mathrm{H}_{34} \mathrm{O}_{2}$ & $2462-85-3$ & 294 & 96 & Nist107 \\
\hline 7 & 22.855 & 0.81 & 1.17 & 3.00 & $\begin{array}{l}9,12,15- \\
\text { Octadecatrienoic } \\
\text { acid, methyl } \\
\text { ester, }(\mathrm{Z}, \mathrm{Z}, \mathrm{Z}) \text { - }\end{array}$ & $\mathrm{C}_{19} \mathrm{H}_{32} \mathrm{O}_{2}$ & $301-00-8$ & 292 & 95 & Nist107 \\
\hline 8 & 24.127 & 0.56 & 0.71 & 3.40 & $\begin{array}{l}\text { Eicosanoic acid, } \\
\text { methyl ester }\end{array}$ & $\mathrm{C}_{21} \mathrm{H}_{42} \mathrm{O}_{2}$ & $1120-28-1$ & 326 & 96 & Nist107 \\
\hline 9 & 24.426 & 0.18 & 0.25 & 3.01 & $\begin{array}{l}\text { 11-Eicosenoic } \\
\text { acid, methyl } \\
\text { ester }\end{array}$ & $\mathrm{C}_{21} \mathrm{H}_{40} \mathrm{O}_{2}$ & $3946-08-5$ & 324 & 81 & Nist147 \\
\hline
\end{tabular}

According to above results showed that total eleven type fatty acid present in n-hexane extract of $G$. tenax. It was mainly found to be in order of 9, 12-Octadecadienoic $(48.50 \%)>9$-Octadecenoic acid $(32.02 \%)>$ Hexadecanoic acid $(11.48 \%)>$ Octadecanoic acid $(6.20 \%)>9,12$, 15- Octadecatrienoic acid $(0.81 \%)>$ Eicosanoic acid $(0.56 \%)>9$-Hexadecenoic acid $(0.19 \%)>11$-Eicosenoic acid $(0.18 \%)$ and Methyl tetradecanoate $(0.06 \%)$.

Table3. Importance of Bioactive compound of hexane extract of leaf of A. pannosum G. tenax

\begin{tabular}{|c|c|c|c|c|}
\hline $\begin{array}{l}\text { Sr. } \\
\text { No. }\end{array}$ & Fatty acid name & Nature & Importance & $\begin{array}{l}\text { Refer } \\
\text { ence }\end{array}$ \\
\hline 1 & $\begin{array}{l}\text { Octanoic acid, } \\
\text { methyl ester }\end{array}$ & Caprylic acid & Candidicide, Flavor, Fungicide, Perfumery, Pesticide & 14 \\
\hline 2 & $\begin{array}{l}\text { Tridecanoic acid, } \\
\text { methyl ester }\end{array}$ & Tridecylic acid & $\begin{array}{c}\text { Antioxidant, Cancer Preventive, Cosmetic, } \\
\text { Hypercholesterolemic, Nematicide, Flavour Ingredient } \\
\text { And Lubricant }\end{array}$ & 15 \\
\hline 3 & $\begin{array}{c}\text { Methyl } \\
\text { tetradecanoate }\end{array}$ & $\begin{array}{l}\text { Myristic acid } \\
\text { ester }\end{array}$ & $\begin{array}{c}\text { Antioxidant, Cancer-preventive, Hypercholesterolemic, } \\
\text { Lubricant, Nematicide }\end{array}$ & 16 \\
\hline 4 & $\begin{array}{l}\text { Hexadecanoic acid, } \\
\text { methyl ester }\end{array}$ & Palmitic acid & $\begin{array}{l}\text { Antiandrogenic, Nematicide, pesticide, } \\
\text { Hypocholesterolemic Hemolytic, } \\
\text { Flavor, Antioxidant, Lubricant, Anti-inflammatory, 5- } \\
\text { Alphareductase inhibitor, Soap, mosquito larvicide }\end{array}$ & $\begin{array}{l}17, \\
18, \\
19,\end{array}$ \\
\hline 5 & $\begin{array}{l}\text { 9-Hexadecenoic } \\
\text { acid, methyl ester, } \\
(\mathbf{Z}) \text { - }\end{array}$ & Palmitoleic acid & $\begin{array}{l}\text { Effects of the permeability and partition of ions into 1, } \\
\text { 2-dimyristoyl-sn-glycero-3- phosphocholine bilayer at } \\
\text { the main phase transition }\end{array}$ & 16 \\
\hline 6 & $\begin{array}{l}\text { Octadecanoic acid, } \\
\text { methyl ester }\end{array}$ & Stearic acid & $\begin{array}{c}\text { Antimicrobial activity, Cosmetic, Flavor, Hypo } \\
\text { cholesterolemic, Lubricant, Perfumery, Propecic, } \\
\text { Suppository }\end{array}$ & 20 \\
\hline 7 & $\begin{array}{l}\text { 9-Octadecenoic } \\
\text { acid (Z)-, methyl } \\
\text { ester }\end{array}$ & Oleic acid & $\begin{array}{l}\text { Anti-Inflammatory, Antiandrogenic, Cancer Preventive } \\
\text {, Dermatitigenic, Hypocholesterolemic, 5- Alpha } \\
\text { reductase inhibitor, Anemiagenic, Insectifuge, } \\
\text { Cosmetic, Flavour, Hypo Cholesterolemic, Lubricant, } \\
\text { Perfumery, Propecic, Suppository }\end{array}$ & 14,15 \\
\hline
\end{tabular}


Soxhtherm Extraction, Isolation and Identification of Fatty Acids Present in the Hexane Extract of Abutilon Pannosum and Grewia Tenax Using Gas Chromatography-Mass Spectrometry

\begin{tabular}{|l|c|c|c|c|}
\hline $\mathbf{8}$ & $\begin{array}{c}\text { 9,12- } \\
\text { Octadecadienoic } \\
\text { acid, methyl ester }\end{array}$ & $\begin{array}{c}\text { Linoleic acid or } \\
\text { Polynoic acid }\end{array}$ & $\begin{array}{c}\text { Hepatoprotective, Antihistaminic, } \\
\text { Hypocholesterolemic, Antieczemic, Anti-Inflammatory, } \\
\text { Antiandrogenic, Cancer Preventive, Dermatitigenic, } \\
\text { Irritant, Antileukotriene-D4, Anti-Cancer }\end{array}$ & 2,22 \\
\hline 9 & $\begin{array}{c}\text { 9,12,15- } \\
\text { Octadecatrienoic } \\
\text { acid, methyl ester, } \\
(\mathbf{Z , Z , Z ) - ~}\end{array}$ & $\begin{array}{c}\text { Polyenoic fatty } \\
\text { acid }\end{array}$ & $\begin{array}{c}\text { Antiinflammatory, Hypocholesterolemic, Cancer } \\
\text { preventive, Hepatoprotective, Nematicide, Insectifuge, } \\
\text { Antihistaminic, Antiacne, Antiarthritic, Anticoronary, } \\
\text { Antieczemic, Antiacne, 5-Alpha reductase inhibitor } \\
\text { Antiandrogenic }\end{array}$ & 2 21, 22 \\
\hline 10 & $\begin{array}{c}\text { Eicosanoic acid, } \\
\text { methyl ester }\end{array}$ & Arachidic acid & Alpha-glucosidase inhibitors & 23 \\
\hline 11 & $\begin{array}{c}\text { 11-Eicosenoic acid, } \\
\text { methyl ester }\end{array}$ & Gondoic acid & Antioxidant, Pesticide, Nematicide & 24 \\
\hline
\end{tabular}

Fatty acids are important bio compounds which take part in complex metabolic pathways, it having major biological roles. It is use full to construct and repair cell membranes enabling the cells to obtain optimum nutrition and expel harmful waste products and also support the cardiovascular, reproductive, immune and nervous systems, is the production of prostaglandins. These regulate body functions such as heart rate, blood pressure, blood clotting, fertility and play a role in immune system by regulating inflammation. ${ }^{[8-12]}$ The analysis of fatty acid from $A$. pannosum and G. tenax by GC/MS showed that it contains various bioactive constituents. In two species presented fatty acid component and its importance were showed above tables. In the present study, except 9, 12-Octadecadienoic and Octadecanoic acid, all the fatty acid contents in A. pannosum was found higher than the G. tenax. Both plant are very important source of fatty acid. In both plants nine same types of fatty acids were present. They are 9, 12-Octadecadienoic, 9-Octadecenoic acid, Hexadecanoic acid, Octadecanoic acid, 9, 12, 15-Octadecatrienoic acid, Eicosanoic acid, 9-Hexadecenoic acid, 11-Eicosenoic acid, Methyl tetradecanoate, Octanoic acid and Tridecanoic acid. But the concentration has been arrived different. The concentration effects on biological activity of component.

\section{CONCLUSION}

In the present investigation higher amount bioactive compound have been identified from n-hexane extract of A. pannosum (11 types of fatty acid) and $G$. tenax (9 types of fatty acid) by Gas Chromatogram-Mass spectrometry (GC-MS) analysis. The presence of various bioactive compounds in both species proved the medicinal importance. In both plants nine types of bioactive compounds were found same. In the present study, except 9, 12-Octadecadienoic and Octadecanoic acid, all the fatty acid contents in A. pannosum was found higher than the G. tenax. Though, further studies might be utilized for the development of traditional medicines and further investigation needs to elute novel active compounds from the medicinal plants which may be create a new way to treat many incurable diseases.

\section{ACKNOWLEDGMENT}

We would like to thank the KSKV Kachchh University (Bhuj), Department of Chemistry to permit for this work. We would also thank to Junagadh Agriculture Food Testing Laboratory for providing facility for this work and guidance.

\section{REFERENCES}

[1] Hill A.F., (1952) Economic Botany. A textbook of useful plants and plant products. $2^{\text {nd }}$ edition McGraw Hill Book Company. Inc. New York

[2] Sriranmsridharan, (2011) GC-MS Study and phytochemical profiling of Mimosa pudica linn. Journal of Pharmacy Research, 4(3): 741-742.

[3] Ananatharaj, M. Venkatesalu, V. Chandrasekaran, M. Sivasankari, S. (2004) Screening of fatty acid methyl esters of marine algae for antibacterial activity. Seaweed Res. Uti., 26: 87-92.

[4] Anup A. Arbat (2012) pharmacognostic studies of stem of abutilon pannosum (forst f.). Bioscience Discovery, 3(3): 317-320.

[5] Abd Elmuti, O. M. (1991) Biochemical and Nutritional Evaluation of Famine Foods of the Sudan; PhD thesis; University of Khartoum Sudan Nov. 7. 
Soxhtherm Extraction, Isolation and Identification of Fatty Acids Present in the Hexane Extract of Abutilon Pannosum and Grewia Tenax Using Gas Chromatography-Mass Spectrometry

[6] Ronald Hites, A. 1997. Gas Chromatography Mass Spectroscopy: Handbook of Instrumental Techniques for Analytical Chemistry, 609-611.

[7] Chen S. H. and Chuang Y. (2002) Analysis of fatty acids by column liquid chromatography. J., Anal. Chim. Acta 465: 145-155, doi./10.1016/S0003-2670(02)00095-8

[8] Connor WE (2000) Importance of n-3 fatty acids in health and disease. Am J Clin Nutr 71: 171.

[9] Tapiero H, Ba GN, Couvreur P, Tew KD (2002) Polyunsaturated fatty acids (PUFA) and eicosanoids in human health and pathologies. Alternative Med Rev, 56: 215-222.

[10] Torres-Duarte AP, Vanderhoek JY (2003) Conjugated linoleic acid exhibits stimulatory and inhibitory effects on prostanoid production in human endothelial cells and platelets. Biochem Biophys Acta Mol Cell Res 69:1640-1647. doi:10.1016/S0167-4889(03)00005-3

[11] Das U (2005) Long-chain polyunsaturated fatty acids, endothelial lipase and atherosclerosis. Prostaglandins, Leukotrienes and Essential Fatty Acids 72 (2005) 173-179. doi:10.1016/ j.plefa. 2004.10.016

[12] Korotkova M, Strandvik B (2000) Essential fatty acid deficiency affects the fatty acid composition of the rat small intestinal and colonic mucosa differently. Biochimica et Biophysica Acta Mol Cell Biol Lipids 1487: 319-325. doi:10.1016/s. 1388 - 1981 ( 00 ) 00121 - 9

[13] Viorica M.P, Alexandra G., Diana N. R., Delia D., Camelia M., Despina B. and Constantin M. (2012). Journal of Agroalimentary Processes and Technologies, 18 (2), 136-140

[14] Dr. Duke's (2012) Phytochemical and Ethnobotanical Databases, doi:10.15482/USDA.ADC/1239279

[15] Owolabi M.A., Coker H.A.B. and Jaja S.I., (2011) Bioactivity of the phytoconstituents of the leaves of Persea americana. J. Med. Plants Res. 4(12): 1130-1135. doi:10.5897/JMPR09.429

[16] Markkas N and Govindharajalu M. (2015). Determination of Phytocomponents in The Methanolic Extract of Mollugo cerviana by GC-MS Analysis. International Journal of Research in Biological sciences. 5(4): 26-29.

[17] Kala, S.M.J., Balasubramanian, T., Tresina Soris, P and Mohan, V.R. (2011). GC-MS determination of bioactive components of Eugenia singampattiana Bedd. Int. J. ChemTech Res. 3: 1534-1537.

[18] Aparna, V.; Dileep, K.V.; Mandal, P.K.; Karthe, P.; Sadasivan, C.; Haridas, M. (2012) Anti-Inflammatory Property of n-Hexadecanoic Acid: Structural Evidence and Kinetic Assessment. Chem. Biol. Drug Des. 80: 434-439. doi: 10.1111/j.1747-0285.2012.01418.x

[19] Kumar, P.P.; Kumaravel, S.; Lalitha, C. (2010) Screening of antioxidant activity, total phenolics and GCMS study of Vitex negundo. Afr. J. Biochem. Res., 4: 191-195.

[20] Rahuman, A.A.; Gopalakrishnan, G.; Ghouse, B.S.; Arumugam, S.; Himalayan, B. (2000) Effect of Feronia limonia on mosquito larvae. Fitoterapia, 71: 553-555.

[21] Ambikapathy, V., Mahalingam, R. and Panneerselvam, A. (2011). GC-MS Determination of Bioactive compounds of Enicostemma littorale (Blume). Asian J. Plant Science and Research, 1 (4):56-60

[22] Abirami, P. and Rajendran, A. (2011). GC-MS Analysis of Tribulus terrestris. Asian J.of Plant Science and Research, 1 (4):13- 16.

[23] A. Elaiyaraja and G. Chandramohan (2015) GC-MS Studies of Crinum defixum Ker-Gawler Leaves. Int. J. Chem, Pharm, Sci (IJCPS), 3(9): 1987-1992.

[24] Amala, K. Saraswathy, A., Amerjothy, S. (2013) GC-MS analysis of N-Hexane extract of Epaltes divaricate (L.) Cass. Journal of Pharmacology and Phytochemistry, 2(1): 33-35.

Citation: M. Aadesariya et al. , "Soxhtherm Extraction, Isolation and Identification of Fatty Acids Present in the Hexane Extract of Abutilon Pannosum and Grewia Tenax Using Gas Chromatography-Mass Spectrometry", International Journal of Advanced Research in Chemical Science (IJARCS), vol. 4, no. 10, pp. 26-34, 2017. http://dx.doi.org/10.20431/2349-0403.0410003

Copyright: (C) 2017 Authors. This is an open-access article distributed under the terms of the Creative Commons Attribution License, which permits unrestricted use, distribution, and reproduction in any medium, provided the original author and source are credited. 